\title{
BIOGRAFÍA DE GASPAR SENTIÑÓN CERDAÑA: DATOS Y ENIGMAS DE UN INTRODUCTOR DE LA MEDICINA INTERNACIONAL EN LA ESPAÑA DE LA RESTAURACIÓN
}

\author{
José Vte. Martí Boscà \\ Unidad de Salud Laboral, \\ Dirección General de Salud Pública, Valencia.
}

\section{RESUMEN}

La biografía se centra en las tres principales actuaciones públicas de Sentiñón: el internacionalismo anarquista, el librepensamiento y la introducción de la medicina internacional en la España de la Restauración. Se han aportado algunos datos necesarios para conocer aspectos confusos de su vida: sus diferencias ideológicas con la evolución del internacionalismo español, las causas de su encarcelamiento, aspectos profesionales y personales, aunque es un personaje que, pese a la importancia de sus actuaciones públicas, intentó siempre permanecer inadvertido.

\section{SUMMARY}

The biography focuses on Sentiñon's three main public spheres of action: anarchist internationalism, freethinking and the introduction of international medicine to Restoration Spain. Certain data required for learning about more confusing aspects of his life has been included, such as details of his ideological differences with the evolution of Spanish internationalism, the causes of his impresonment, professional and personal sides, though we are studying a figure who always tried to go unnoticed, in spite of the importance of his public role.

\section{INTRODUCCIÓN}

Luis Carlos Gaspar Sentiñón Cerdaña es una figura singular de la España de la Restauración. Por una parte, contribuyó de modo decisivo a la difusión de la información médica internacional a través de una serie asombrosamente amplia de traducciones, resúmenes, noticias, adaptaciones y síntesis. En ella demostró un inusual dominio de idiomas tan variados como el alemán, ruso, francés, inglés, italiano, grie- 
go y rumano, así como una sólida preparación médica y científica ${ }^{1}$. Por otro lado, fue un destacado militante del movimiento proletario, que participó en la constitución del anarquismo en directa relación con Bakunin, así como un activo seguidor de las corrientes librepensadoras más radicales ${ }^{2}$.

En contraste con su notoriedad en ambas vertientes, su biografía está llena de importantes lagunas e imprecisiones, en especial en lo referente a su origen, infancia y juventud y a su formación, que han sido motivo de suposiciones no confirmadas o incluso desmentidas por las fuentes. Por razones que nos son desconocidas, el propio Sentiñón se preocupó de ocultar su auténtica personalidad, hasta el punto de que, como vamos a ver, solamente confió a Bakunin el llamado «misterio de su vida».

El objetivo del presente artículo, que corresponde a una línea de estudio dedicada a Sentiñón desde una doble perspectiva histórico-médica e histórico-política, consiste en exponer ordenadamente la información que acerca de su trayectoria biográfica ofrecen las fuentes hasta ahora localizadas. No se ocupa, por el contrario, del contenido de sus numerosísimas publicaciones, de las que únicamente se citan las más importantes con la intención de situarlas en las sucesivas etapas de su vida. En posteriores investigaciones, se intentará contribuir al análisis de dicho contenido.

\section{SU ORIGEN Y SUS AÑOS DE MILITANTE ANARQUISTA (¿1835?-1871)}

La única fuente que informa del nacimiento de Sentiñón es su certificado de defunción, en el que, entre otros datos, figura con el nombre de «Gaspar Sentiñón y Cerdaña», natural de Barcelona y fallecido en esta misma ciudad el 12 de diciembre de 1902, a los 67 años de edad ${ }^{3}$. Por los motivos antedichos no puede, sin embargo, considerarse un dato indiscutible ni siquiera el nombre. Con diverso fundamento, se ha atribuido a su padre la nacionalidad austríaca ${ }^{4}$ y a su madre, la rusa ${ }^{5}$. En cualquier caso, consta que residió en Alemania desde los seis años de edad ${ }^{6}$, pero se ignoran los lugares y centros en los que adquirió su formación. Generalmente se afirma que

1 El inicio de esta investigación sobre la biografía se expone en nuestra tesis doctoral, donde presentamos el repertorio de sus publicaciones y la bibliometría de su obra médica. La tesis ha sido realizada bajo la dirección del profesor José $\mathrm{M}^{\mathrm{a}} \mathrm{López} \mathrm{Piñero,} \mathrm{a} \mathrm{quien} \mathrm{agradecemos} \mathrm{especialmente} \mathrm{los} \mathrm{comentarios}$ y sugerencias al borrador de este artículo.

${ }^{2}$ Los principales trabajos dedicados a Sentiñón desde la Historia de la Medicina son: CoRBELLACAlbet (1969); CALBET-CORBElla (1981-1983), v. 3, p. 96-97; BuJOSA, en: LóPEZ PIÑERO et al. (1983), v 2, p. 316-317 y BRETAU (1987).

3 Registro Civil, Barcelona. Sección Tercera, Libro 96 (6), inscripción 1970.

4 Arthur Lehning en las notas de su edición crítica de BAKUNIN (1973-1982), v. 7, p. 422, n. 125.

5 CARRERAS (1979), p. 40 y 50.

6 LiMA (1892), p. 319, que se basa, para esta y otras afirmaciones sobre el movimiento obrero en España, en los informes enviados por Juan Salas Antón desde Barcelona. 
cursó medicina en la Universidad de Viena ${ }^{7}$, pero en la documentación existente en el Archivo de esta institución no aparece registrado en el periodo 1853-1868, al menos con los nombres y apellidos con que ha pasado a la posteridad.

El primer dato firme conocido es que el 7 de febrero de 1869 escribió en la localidad prusiana de Lautenburg el artículo titulado «Allgemeine Bildung», que apareció en la revista suiza Vereinigten Staaten von Europa, que publicaba la Liga por la Paz y la Libertad, asociación de orientación europeísta. En él propugnó una ortografía simplificada del idioma alemán, lo que refleja ya su interés por las cuestiones lingüísticas.

La siguiente información disponible corresponde al inicio de su vinculación a la Internacional, período en el que el destacado papel que ejerció nos permite, de forma inversa a la etapa anterior, un detallado seguimiento de sus actividades. En la sesión del Consejo General de la Asociación Internacional de los Trabajadores (AIT), celebrada el 15 de junio de 1869 en su sede londinense, se leyó una carta suya enviada desde Prusia Oriental, en la que solicitaba información acerca del secretario de la Sección de Barcelona, ya que había decidido volver a España ${ }^{8}$.

Probablemente requerido por Bakunin, al que aún no conocía personalmente ${ }^{9}$, acudió a las reuniones previas al Cuarto Congreso de la Internacional, que se iba a celebrar en Basilea en septiembre de ese año. Meses antes, la Alianza Internacional de la Democracia Socialista, el grupo de partidarios del ruso, había sido aceptada en la AIT como una sección local de Ginebra, pero no como una organización internacional con afiliados propios.

Los obreros catalanes que se agrupaban en el Centro Federal de las Sociedades Obreras de Barcelona enviaron al Congreso de Basilea a su secretario, Rafael Farga Pellicer, para fortalecer las relaciones con la AIT, aunque éste ya se había manifestado como partidario de las ideas de Bakunin ${ }^{10}$.

El 28 de agosto, en Ginebra, Sentiñón y Farga asistieron a la reunión del comité de la Alianza y fueron recibidos afectuosamente por Bakunin ${ }^{11}$. Sentiñón, al tiempo que fue admitido como miembro de esta organización, se ofreció al delegado barcelonés para representar a los obreros del Centro Federal. Al día siguiente, se celebró la asamblea de la Alianza para preparar el Congreso, en la que Sentiñón fue elegido como representante de esta sección ginebrina ${ }^{12}$. Su formación científica, sus conoci-

\footnotetext{
7 En Guillaume (1985), p. 242, y posterirmente en el resto de trabajos sobre Sentiñón.

8 The General Council of the first International, 1868-1870: Minutes, p. 112; citado y traducido por ARBELOA (1972), p. 60. Hemos consultado el mismo texto de la sesión en la versión en idioma francés: CONSEIL GÉNÉRAL (1974), p. 94.

9 Así lo afirma el anarquista ruso, véase BAKUNIN (1973-1982), v. 1, p. 212.

10 Ver su carta a Bakunin de 1 de agosto de 1869, reproducida por NETTLAU (1925), p. 45-47.

${ }^{11}$ La Federación, 8 (19 de septiembre de 1896).

12 Guillaume (1985), v. 1, p. 188.
} 
mientos políticos y el dominio de idiomas hacen especialmente atractiva la figura del español, que recibió esta doble delegación.

El Cuarto Congreso de la AIT ${ }^{13}$, celebrado en Basilea desde el 5 al 12 de septiembre, reunió a delegados de las sociedades obreras francesas, suizas, belgas, inglesas, austríacas, italianas, norteamericanas y españolas. Tuvo gran importancia para los trabajadores españoles, ya que supuso el inicio de su integración en la organización internacionalista.

La participación de Sentiñón en este Congreso no pasó desapercibida. Así, con Farga, se encargó de la secretaría de lengua española, al tiempo que formaba parte de la comisión de recepción de comunicados. En las ponencias para elevar al pleno del Congreso, se incorporó a tres de las cinco comisiones: las dedicadas a la propiedad de la tierra, al crédito mutual y a la educación integral. En cuanto a las votaciones, se adscribió al grupo denominado antiautoritario, encabezado por Bakunin, frente a las tesis del Consejo General, liderado por Carlos Marx, que no acudió al Congreso.

Debemos resaltar tres puntos de su participación en el Congreso de Basilea. En primer término, el «Informe de los delegados del Centro Federal de las Sociedades Obreras de Barcelona» ${ }^{14}$, leído en el Congreso por Farga y firmado conjuntamente con Sentinón; en él se expuso la situación del asociacionismo obrero en España tras la caída de Isabel II. En segundo lugar, su papel en el juicio de honor solicitado por Bakunin a causa de los ataques que había recibido de los socialdemócratas alemanes, acusándolo de ser agente del gobierno ruso. Neumayer, Palix, Robert, De Paepe y Sentiñón ${ }^{15}$ fueron elegidos por el ruso como jurado. Se proclamó la inocencia de Bakunin, que recibió las disculpas del dirigente alemán Liebknecht como inductor de los hechos. Como tercer tema, y probablemente el más importante, debemos destacar su participación en la comisión sobre la educación integral, junto al pedagogo Paul Robin; si bien esta ponencia no pudo ser leída en el pleno por falta de tiempo, sus debates fueron importantes para la posterior orientación de los internacionalistas españoles sobre la educación ${ }^{16}$. Sentiñón actuó como transmisor inicial del pensamiento pedagógico de Robin.

Una vez acabado el Congreso, Farga y Sentiñón permanecieron algún tiempo en Suiza con Bakunin, formando parte del proyecto de éste de crear un grupo revolucionario formado por miembros escogidos de las secciones de Italia, España, Francia y Suiza, grupo que se conoce como Alianza $«$ secreta» ${ }^{17}$, de la que correspondió a Sentiñón el número 60 de asociado $^{18}$.

\footnotetext{
13 Los textos del Congreso de Basilea en FREYMOND (1973), v. 2, p. 5-174.

14 Ibidem, p. 50-54.

15 GuILlaUME (1985), v. 1, p. 212

16 HERNÁNDEZ (1992), p. 218-219.

17 LIDA (1972), p. 155.

18 Ibidem, p. $155-156$ y n. 102.
} 
Tras informar a la Sección de Ginebra como su delegado en el Congreso, Sentiñón volvió a Barcelona con la idea de plasmar su pensamiento radicalmente antirreligioso mediante la constitución de una sociedad librepensadora.

El librepensamiento moderno español es hijo directo de la revolución de la Gloriosa ${ }^{19}$ y en él confluyeron tanto el sector más progresista de la masonería como los republicanos federalistas y los internacionalistas, al inicio íntimamente unidos. Fruto de la actividad de Sentiñón, se inició la creación de una sociedad de librepensadores que, como él mismo comunicó a la prensa internacionalista, tanto de Barcelona ${ }^{20}$ como de Ginebra ${ }^{21}$, adoptó provisionalmente los reglamentos de la sociedad homóloga de Marsella.

Desde Barcelona viajó a Alemania y Bélgica con objeto de estudiar la adquisición de armas para un posible levantamiento obrero ${ }^{22}$. Luego regresó a Neuchâtel, a casa del compañero de militancia y gran amigo de Bakunin, James Guillaume; éste, que ha recogido en su obra la evolución de la AIT desde su adscripción al grupo antiautoritario, nos explica la gran intimidad que se produjo entre Bakunin y Sentiñón, lo que llevó al español, siempre muy reservado, a confiar al ruso el «misterio de su vida» ${ }^{23}$, enigma cuyo argumento Guillaume no conoció pero del que hay otro testimonio entre los internacionalistas más cercanos a Bakunin ${ }^{24}$. El contenido de este misterio permanece sin desvelar, aunque lo podemos suponer relacionado con su origen y lo inusual de su apellido.

En Suiza, Sentiñón participó en algunas reuniones de las secciones locales de la AIT, como la Asamblea de Locle celebrada el 23 de noviembre ${ }^{25}$. Posteriormente, tras visitar a Bakunin y Fanelli en Locarno, regresó a España desde Ginebra, pasando por Lyon y Marsella, cuyas agrupaciones internacionalistas quería conocer ${ }^{26}$.

En los pocos meses que transcurrieron desde finales de agosto hasta diciembre de 1869, Sentiñón se integró en el grupo internacionalista más próximo a Bakunin, cuyo pensamiento y amistad compartía plenamente. El líder ruso, muy aficionado a las organizaciones secretas, utilizaba en su correspondencia los nombres en clave de «Monsieur E F» y «François» para referirse al español, de forma similar a lo que

19 SÀNCHEZ (1990), p. 99-103.

${ }^{20}$ La Federación, 16 (14 de noviembre de 1869).

${ }^{21}$ L'Egalité, 44 (20 de noviembre de 1869).

22 GuILlaume (1985), v. 1, p. 242 y la carta de Sentiñón al internacionalista Johann Philipp Becker (8 de diciembre de 1869), traducida por NeTTLAU (1930), p. 14-15.

23 Guillaume (1985), v. 1, p. 242.

24 RICHARD (1896), p. 124.

${ }^{25}$ La Federación, 20 (13 de diciembre de 1869), que reproduce la nota del periódico internacionalista de Locle Le Progrès, 24 (27 de noviembre de 1869).

${ }^{26}$ La reconstrucción de este periodo ha sido realizada con detalle por MADRID (1995). 
hacía con sus colaboradores más íntimos. Una prueba de la estrecha relación son las más de veinte cartas cruzadas entre ambos, de las que sólo algunas se conservan ${ }^{27}$.

En diciembre, a su regreso a Barcelona, Sentiñón volcó su gran capacidad de trabajo en dos líneas confluentes: el desarrollo de la Internacional en Cataluña y la constitución de una sociedad de librepensadores.

El organismo coordinador de las sociedades obreras de Barcelona, el Centro Federal, no tenía una definición política anarquista, sino de carácter republicano federal; por ello, la actividad de un pequeño grupo encabezado por Farga y Sentiñón tuvo como objetivo lograr esa evolución ${ }^{28}$. Este grupo utilizó dos instrumentos: la prensa y las asambleas. En La Federación, el órgano de prensa del Centro Federal que pronto se transformó en el periódico más importante del internacionalismo hispano, se reprodujeron escritos de Bakunin, probablemente traducidos por Sentiñón, así como noticias y comentarios publicados en la prensa internacionalista europea, con la que mantuvo habituales intercambios.

El 4 de enero de 1870, en la misma reunión en que Farga y Sentiñón leyeron su informe sobre el Congreso de Basilea, el Centro Federal pasó a denominarse Centro Local de la Asociación Internacional de los Trabajadores ${ }^{29}$. Farga y Sentiñón informaban periódicamente a Bakunin, quien en sus escritos a otros miembros de la Alianza expresaba su plena satisfacción por la actuación de los dos españoles ${ }^{30}$.

Al mismo tiempo, Sentiñón participaba en el desarrollo de la organización de los librepensadores de Barcelona. En diciembre de 1869 se publicó otra nota suya ${ }^{31}$ con una dura respuesta a los comentarios del periódico de Tortosa La Voz de la Patria sobre el proyecto de sociedad librepensadora. Pocos días después, en enero de 1870, tuvo lugar una reunión en el teatro Novedades ${ }^{32}$, en la que se repartieron ejemplares de los estatutos de la futura sociedad, que se aprobaron en la siguiente reunión del 6 de febrero, como confirma un conciso escrito en la prensa internacionalista ${ }^{33}$, siempre atenta al desarrollo del grupo librepensador. La sociedad resultante recibió el nombre de Asociación Libre-Pensadora de Barcelona que, aunque no era la primera en crearse, fue la más destacada de su época ${ }^{34}$. Su órgano de prensa, el semanario $L a$ Humanidad, salió a la calle el último día de 1870. Durante el primer año de existencia de esta revista, Sentiñón desarrolló en ella una importante tarea, ya que es autor

\footnotetext{
27 Una relación epistolar entre Sentiñón y los principales internacionalistas, con sus respectivas referencias, en MARTí (1997), p. 86-89.

28 Véase LORENZO (1974), p. 51-57.

29 NeTTLAU (1969), p. 76.

30 Ver, entre otras, la carta de Bakunin a Albert Richard de 7 de febrero de 1870, en BAKUNIN (1973-1982), v. 7, p. 271-273.

31 La Federación, 20 (12 de diciembre de 1869).

32 VilanOU (1978), p. 94.

33 La Federación, 29 (13 de febrero de 1870).

34 Vilanou (1978), p. 92-97.
} 
de las breves noticias de carácter antirreligioso, científico o de divulgación médica que encontramos en la sección «Crónica». Igualmente, publicó con su firma tres amplios artículos de contenido filosófico.

Paralelamente al progreso de estas actuaciones, Sentiñón mantuvo correspondencia con los principales líderes del internacionalismo europeo, lo que le permitió estar informado y participar en los debates de diferentes secciones de la AIT, como en el asunto Liebknecht-Schwetzer ${ }^{35}$, cuyo tratamiento por los periódicos Le Progrés y L'Egalité provocó la «Comunicación privada» del Consejo General de Londres.

También, poco más tarde, el 31 de marzo de 1870, él y Farga envían una carta al Congreso de la Sección de la AIT de la Suiza romanche ${ }^{36}$. La escisión de ésta repercutirá en la Sección española, pues ambos, probablemente influenciados por lo sucedido en la Sección suiza ${ }^{37}$, crearon en abril un pequeño grupo dentro de la española, similar al constituido como la Alianza «secreta» de Ginebra, al que dieron el mismo nombre, Alianza de la Democracia Socialista, pero con reglamento y programa propios; el objetivo inmediato era preparar un congreso de las sociedades obreras, atrayéndolas a la Internacional. Posteriormente, este grupo se desarrolló en otras ciudades españolas (Palma de Mallorca, Valencia, Sevilla, Madrid,... $)^{38}$. Con la formación de esta organización Sentiñón participó activamente en todos los grupos de inspiración bakuninista creados en la Internacional: la Alianza «pública» de Ginebra, la Alianza «secreta» de Ginebra y la Alianza «secreta» española.

El primer Congreso Obrero Español, la esperada reunión de las sociedades obreras celebrada en el Teatro Circo, de Barcelona, del 19 al 26 de junio $^{39}$, supuso un gran avance para los internacionalistas españoles, mayoritariamente adscritos a la tendencia antiautoritaria. Su alianza con los sindicalistas apolíticos derrotó tanto a los cooperativistas como a los obreros partidarios de la acción política.

Sentiñón asistió al Congreso como delegado de los marineros de Baleares y de los cerrajeros, impresores, mecánicos y Sección Central de Cádiz, según la costumbre de representar a sociedades a las que no se pertenecía. Su participación en los debates no se correspondió con el papel que desarrollaba en la Sección española de la Internacional ni en el propio núcleo bakuninista de la AIT: sólo votó en dos sesiones, las relativas a la resistencia obrera y a la cooperación, y formó parte del dictamen sobre la organización social de los trabajadores, pero no estuvo en su votación. Ni siquiera el tema estrella del Congreso, la Internacional y la política, que ocupó cuatro sesiones, contó con su participación ni con su voto, aunque durante su desarrollo_presentó,

35 Comentado por Vilanou en su introducción a RoBIN (1981), p. 13.

36 Reproducida por GuILlaUme (1985), v. 2, p. 27.

37 Suscribimos esta hipótesis planteada por MADRID (1995), p. 44 y n. 45.

38 TERMES (1977), p. 151.

${ }^{39}$ El seguimiento del Congreso en los suplementos de La Federación, desde el 19 de junio al 7 de agosto de 1870. Trascripción con notas en ARBELOA (1972). 
con González Morago y Francisco Tomás, una proposición urgente de protesta por la prohibición del congreso obrero de Ruán, que fue aprobada por unanimidad.

En julio comenzó la guerra franco-prusiana y Bakunin, siempre atento a los movimientos revolucionarios, creyó ver nuevas posibilidades de acción, por lo que escribió a sus íntimos para que acudiesen a Francia con objeto de impulsar las esperadas revueltas populares. Entre los convocados estaba Sentiñón. Cuando éste llegó a Lyon, el movimiento había fracasado y Bakunin había partido hacia Marsella con intención de pasar a Barcelona, ya que confiaba en la capacidad revolucionaria de los obreros españoles; dado el resultado, el ruso envió una emotiva carta ${ }^{40}$ a Sentiñón, aconsejándole que regresase. El español llegó a Marsella el 25 de octubre, el día siguiente a la precipitada huida de Bakunin, y participó en el movimiento local, que también fue un fracaso ${ }^{41}$, por lo que volvió a Barcelona.

El desenlace de esta breve experiencia revolucionaria y, sobre todo, la posterior derrota y represión de la Comuna de París, incluyendo la tortura y fusilamiento de su amigo Eugène Varlin -destacado sindicalista francés con el que había mantenido contacto epistolar desde el Congreso de Basilea-, debieron influir en su valoración de la táctica insurreccional. Así, en las cartas que escribe en abril y mayo de 1871 se puede observar su opinión negativa sobre la incidencia de las frecuentes huelgas en el desarrollo de la Internacional, la consideración de que un levantamiento internacionalista en Cataluña sólo favorecería a los carlistas y el temor a una represión generalizada contra la $\mathrm{AIT}^{42}$. De hecho, Sagasta, ministro de Gobernación, anunció a finales de mayo en las Cortes medidas represivas contra la Internacional, especialmente en Barcelona ${ }^{43}$.

En este contexto, el Consejo Federal español de la AIT había decidido trasladarse a Lisboa ${ }^{44}$ y Farga Pellicer, director de La Federación, se escondió. El propio órgano internacionalista, del que Sentiñón era administrador, denunció las acciones del juzgado de distrito contra el periódico ${ }^{45}$. Como cabeza destacada de los internacionalistas, fue detenido en su domicilio en la madrugada del 7 de junio, por orden del gobernador civil, e incomunicado en el cuartelillo de la aduana ${ }^{46}$. El 15 de junio fue trasladado a la cárcel del castillo de Montjuich, de donde salió, sin que se produjese acción judicial alguna, a finales de septiembre del mismo año.

\footnotetext{
40 Reproducida por Nettlau en su introducción a BAKUNIN (1977-86), v. 1, p. 44-45.

41 NeTtLau (1925), p. 62.

42 Especialmente ver su carta reproducida en Der Vorbote, 4 (abril de 1871), transcrita y traducida en MARTí (1997), p. 603-606.

43 ÁlVAREZ (1971), p. 41-48 y 87-99.

44 Ver el acta de la reunión transcrita por SECO (1969), p. 62-63.

45 La Federación, 94 (4 de junio de 1871).

46 La Federación, 95 (11 de junio de 1871).
} 
El encarcelamiento de Sentiñón ha estado envuelto en confusiones y errores. Así, se ha atribuido su detención a que había firmado, junto al presidente de la sociedad obrera Las Tres Clases de Vapor, Clemente Bové, el «Manifiesto de algunos partidarios de la Commune a los poderosos de la tierra» ${ }^{47}$. Lo cierto es que este documento no lo firmaron ninguno de los dos, sino los internacionalistas Anselmo Lorenzo, Francisco Mora y Tomás González Morago ${ }^{48}$, de la Sección de Madrid; no era coherente que lo hubiese firmado, por lo que hemos expuesto sobre su evolución ideológica.

Parece ser que su ingreso en prisión le afectó mucho ${ }^{49}$. Desde Montjuich envió una carta al director de La Federación en la que justificaba su detención preventiva y expresaba su divergencia con la dirección de la Internacional en España ${ }^{50}$. También desde la cárcel escribió una carta de protesta a La Crónica de Cataluña $a^{51}$, sobre la circular del ministro francés Jules Favre que aconsejaba a los gobiernos europeos desarrollar acciones contra la AIT. Meses más tarde, explicó en una carta al dirigente socialdemócrata Liebknecht su separación de la Internacional, siempre en clave misteriosa ${ }^{52}$.

DESDE SU ABANDONO DE LA MILITANCIA ANARQUISTA HASTA SU MUERTE (18721902)

Con su encarcelamiento Sentiñón rompió con la militancia anarquista y cesaron sus colaboraciones con el órgano librepensador La Humanidad ${ }^{53}$, que finalizó su existencia con el número 69, ya en 1872 . No obstante, el abandono de la militancia no le libró de verse implicado en la disputa entre marxistas y bakuninistas, que fue especialmente intensa en España por la presencia del yerno de Marx, Paul Lafargue. Así, en julio de 1872, el periódico del grupo marxista La Emancipación ${ }^{54}$ hizo pública una relación de aliancistas, en la que incluyó a Sentiñón, pese a que su aislamiento era bien conocido. En la imprenta de este periódico, Lafargue editó un folleto denominado A los internacionales de la Región Española ${ }^{55}$, en el que nuevamente se le atacaba.

47 El origen de esta versión en GUILlaUME (1985), v. 2, p. 164. MADRID (1995), p. 47, n. 65, explica las causas de la detención de Bové, que sólo coincide con Sentiñón en el traslado a Montjuich.

48 Reproducido y comentado en El Imparcial, (7 de junio de 1871).

49 La Federación, 95 (11 de junio de 1871).

50 La carta no fue publicada por el semanario internacionalista, pero sí por el diario republicano federal La Independencia el 20 de junio de 1871. Reproducida por MADRID (1995), p. 51-52.

51 Esta carta fue publicada por La Federación, 97 (27 de junio de 1871).

52 Reproducida por NETTLAU (1926), p. 327-328. Liebknecht en sus siguientes cartas a Engels (15 de febrero) y Becker (22 de febrero) no disimuló su satisfacción por la marcha de Sentiñón, conociendo su gran vinculación con Bakunin, al tiempo que expresó sus dudas sobre su pasado, llegando a insinuar alguna relación policial; véanse los fragmentos de ambas cartas en RIBAS (1979), p. 228, n 3.

$53 \mathrm{Su}$ última colaboración firmada se publica en el número $1 / 25$, el 24 de junio de 1871 .

54 La Emancipación, 59 (29 de julio de 1872).

55 Reproducido por LIDA (1973), p. 244-266. 
Los agravios continuaron en 1873 al editarse un nuevo folleto, esta vez desde Londres, con el título La Alianza de la Democracia Socialista y la Asociación Internacional de los Trabajadores ${ }^{56}$, firmado por el Consejo General de la AIT y atribuido a Engels, Lafargue, Marx y Outin. Este documento es el colofón a la resolución del Congreso de la AIT en La Haya por la que se expulsó a Bakunin y Guillaume, con la consiguiente escisión de los antiautoritarios.

Sentiñón que, como hemos visto, había representado a la Alianza «pública» de Ginebra en el Congreso de Basilea y participado en la creación de las Alianzas «secretas» de Ginebra y Barcelona, era un ejemplo demasiado valioso en el enfrentamiento entre Marx y Bakunin para ser olvidado, aunque Lafargue conocía su separación de la Internacional, como recordó la prensa de tendencia bakuninista ${ }^{57}$.

El alejamiento de la Internacional llevó a Sentiñón a aproximarse al grupo más izquierdista de los republicanos de Barcelona, constituido en torno al centro El Estado Catalán o Club de los Federalistas, que lideraba Valentí Almirall. En la necrología que a su fallecimiento le dedicó un diario republicano se le atribuía un papel principal en este centro, pero en coherencia con su personalidad «sin que jamás aspirara a obtener representación popular alguna» ${ }^{58}$. Lo que hemos comprobado es que en abril de 1873 los federales de El Estado Catalán publicaron un manifiesto atacando duramente al Comité Republicano de Barcelona por excederse en sus funciones, titulado «El Estado Catalán, centro de los republicanos demócratas federalistas, al público en general y a sus correligionarios en particular»; entre los firmantes figura Sentiñón ${ }^{59}$.

Cuando tras las elecciones se proclamó la República Federal -en el contexto de los levantamientos cantonalistas-, se convocó en Barcelona, en la tarde del 19 de junio de 1873, una manifestación de apoyo a las tropas del batallón de cazadores de Madrid, responsables de la muerte de su coronel en Sagunto. Los amotinados ocuparon el Ayuntamiento con gritos a favor del batallón, de la revolución, del Estado Catalán, del Ayuntamiento autónomo y de la federación ${ }^{60}$. Uno de los más destacados manifestantes era el médico y antiguo compañero de Sentiñón en la Alianza, José García Viñas. Los integrantes de la manifestación, una vez tomado el Ayuntamiento, constituyeron un «Comité de salvación pública» formado por los comandantes de los batallones de voluntarios de la República, delegados del Casino Instructivo, del Círculo de Recreo, de los trabajadores -entre ellos García Viñas- y del Estado Catalán,

56 Reproducido por FREYMOND (1973), v. 2, p. 449-565.

57 La Federación, 155 (4 de agosto de 1872). También se publicó la nota de protesta en los periódicos anarquistas La Razón (Sevilla) y El Condenado (Madrid), pero no en La Emancipación.

58 El Diluvio, 342 (14 de diciembre de 1902). Esta necrología tiene el sugerente título de «Fallecimiento de un sabio».

59 Reproducido por GONZÁLEZ (1896), p. 245-248. TERMES (1977), p. 140, le atribuye su participación en otros manifiestos publicados en el periódico El Estado Federal, órgano del centro.

${ }^{60}$ Diario de Barcelona, 171 (20 de junio de 1873, ediciones de la mañana y de la tarde). 
uno de los cuales era Sentiñón. El día siguiente, tras emitir un comunicado de apoyo al batallón de cazadores, y sin mediar ataques ni refuerzos, el Comité, ya aminorado a «Comisión de vigilancia de la federación y de la democracia», se disolvió.

El abandono de las militancias internacionalista y librepensadora, a las que había dedicado una intensa actividad, permitió aflorar al Sentiñón que desarrolló ampliamente sus facetas de intelectual médico: difusor de la medicina internacional entre los profesionales españoles, traductor de textos médicos y divulgador del higienismo entre la población.

Aunque en su etapa militante había mantenido contacto con la prensa médica, como demuestran sus artículos sobre temas científico-médicos en el órgano librepensador La Humanidad y sus primeras colaboraciones profesionales en La Independencia médica (1871), esta etapa la podemos iniciar con la publicación de sus primeras traducciones, la del Manual completo de terapéutica (1872), del profesor de Viena Kraus, y la de una obra filosófica de especial interés para los anarquistas ${ }^{61}$, Ciencia y naturaleza, recopilación de publicaciones realizada por Ludwing Büchner, en cuyo prólogo, atribuido a Sentiñón ${ }^{62}$, se expone la importancia de la divulgación como tarea revolucionaria del momento.

En su extensa bibliografía, Sentiñón hizo escasas menciones a su propia experiencia profesional y los poquísimos casos de consultas de pacientes que comentaba en sus textos pueden entenderse ajenos al ejercicio de una medicina asistencial continuada. De vida muy reservada, hasta sus propios compañeros, entre los que era muy conocido por sus escritos, dudaron de su experiencia práctica ${ }^{63}$. Su actividad profesional se centró en la enseñanza de idiomas y en el mundo de las publicaciones, tanto como traductor o director de colecciones como comentarista de noticias y congresos médicos. Años después de su fallecimiento, Rodríguez Méndez lo recordaba como «el no médico» ${ }^{64}$, a pesar de los conocimientos en Medicina que atribuía al que propuso llamar «biblioteca-Sentiñón» ${ }^{65}$. Es indudable su elevada formación médica, muy superior a la de un traductor especializado o a la de un profesional de la medicina, como le reconocía el decano de la Facultad de Medicina Giné y Partagás ${ }^{66}$.

Su erudición en lenguas era excepcional, su contemporáneo Salas Antón afirmó que dominaba veintinueve idiomas ${ }^{67}$. De su obra se deduce que traducía, además de los idiomas ya comentados, el latín, sánscrito y griego clásico, con amplios conocimientos del árabe, chino y sueco.

\footnotetext{
61 La Federación lo anunció en sus páginas el 19 de junio de 1873.

62 NúÑ̃Z (1975), p. 38, considera que el prólogo es de Sentiñón.

63 ROVIRA (1884), p. 365.

64 RODRÍGUEZ MÉNDEZ (1919), p. 170. Ver también p. 156.

65 RODRÍGUEZ MÉNDEZ, (1881), p. 211

66 GINÉ (1898), p. 32, n.

67 REVENTÓs (1987), p. 120, n. 7.
} 


\section{JOSÉ VICENTE MARTÍ BOSCÀ}

Sentiñón, con su capacidad de trabajo y su formación, se incorporó a la medicina catalana del último tercio del siglo en un momento especialmente fructífero, en el que destacadas figuras como José de Letamendi, que todavía gozaba de gran prestigio, iban a ser relevadas por la nueva generación médica que encabezaron profesionales como Juan Giné y Partagás, Ignacio Valentí Vivó, Bartolomé Robert Yarzábal o Rafael Rodríguez Méndez.

Aunque Sentiñón se relacionase con todos ellos, su introducción en el ambiente médico barcelonés fue el fruto de su colaboración con Letamendi. Puede parecer paradójico el gran vínculo que existió entre el antiguo internacionalista y el polifacético y controvertido catedrático de anatomía, cuya sistematización especulativa es un eco tardío de la Naturphilosophie idealista romántica ${ }^{68}$, pero tenían varios puntos en común. Letamendi gustaba de expresiones obreristas como el ser socio honorario del Ateneo Catalán de la Clase Obrera desde 1869, centro íntimamente ligado -compartía local y secretario, Farga Pellicer- con el Centro Federal de las Sociedades Obreras, al que Sentiñón había pertenecido de forma destacada. También les unió el interés por la higiene y la divulgación sanitaria, dos líneas de trabajo preferidas de Sentiñón que su relación con Letamendi le permitió desarrollar.

En este equipo, Letamendi era la cabeza visible, mientras que Sentiñón, con su excelente refuerzo teórico y una gran capacidad de trabajo, plasmaba los resultados. Letamendi le denominaba amistosamente «mi ayudante cerebral» ${ }^{69} \mathrm{y} « \mathrm{mi}$ antiguo maestro en asuntos filológicos, lingüísticos y otros más» ${ }^{70}$.

Fruto de esta colaboración fue la edición de dos revistas a principios de 1877. El 7 de enero, La Salud; una semana más tarde, Archivo de la cirugía. La idea era completar la primera, de carácter divulgativo, con Archivo, revista eminentemente científica, de tal forma que la suscripción conjunta resultaba más económica. La trayectoria de ambos semanarios fue diferente: de Archivo de la cirugía, en cuya portada figura Letamendi como director-propietario y Sentiñón como subdirector, sólo conocemos dos números publicados en el mismo ejemplar; La Salud se editó durante dos años y finalizó por el traslado de su director al ocupar cátedra en Madrid.

Por el carácter predominantemente anónimo de las páginas de La Salud es difícil cuantificar la participación de Sentiñón, aunque pocos años después de concluida esta revista Rodríguez Méndez ${ }^{71}$ le atribuyó su redacción casi exclusiva. Ciertamente, volcó su gran capacidad de trabajo en una época en la que, todavía soltero, residía y trabajaba en el piso superior en la casa de Letamendi ${ }^{72}$. El fructífero trabajo sobre

\footnotetext{
68 LÓPEZ PIÑERO (1992), p. 234.

69 SENTIÑón (1897), p. 421.

70 LETAMENDI (1894), v. 1, p. 41.

71 RODRÍGUEZ MÉNDEZ (1881), p. 211.

72 RODRÍGUEZ MÉNDEZ (1919), p. 156.
} 
higiene laboral que desarrolló desde la sección «La higiene del proletario» ya no tuvo continuación en otras revistas en las que participó.

También durante estos años comenzó a colaborar con otras publicaciones médicas. En 1876 con la barcelonesa Revista de ciencias médicas, con la que mantuvo su contribución durante décadas. En 1878 inició su participación en otra revista médica a la que también permaneció vinculado de por vida, Gaceta médica de Cataluña, que desde 1881 se tituló Gaceta médica catalana. Al principio era el responsable de la sección «Meteorología y Demografía», luego continuó con sus traducciones, resúmenes y noticias médicas.

En el mismo año de 1878 finalizó la traducción del libro de Day sobre las cefalalgias, que se publicó dos años después; en febrero de 1879 apareció su firma en otra revista médica con la que colaboró durante décadas, la Revista de medicina y cirugía prácticas, de Madrid. También ese año se distribuyeron dos nuevos libros médicos traducidos del alemán por Sentiñón: el de Niemeyer, relativo a la tos, y el del doctor de Guerard, sobre los dientes; ambos fueron publicados en la «Biblioteca Popular Ilustrada de Higiene y Medicina Domésticas», colección de la que era director para la editorial de Riera.

El año siguiente, 1880, se publicó su primer libro, La digestión y sus tropiezos, en el que trata también de la nutrición, uno de los temas médicos que más le interesaron. Pocos meses después, ya en 1881, inició su colaboración con Anales de obstetricia, ginecopatía y pediatría, revista en la que siguió escribiendo hasta que ésta se integró en la Revista de medicina y cirugía prácticas. Ese año se presentaron dos nuevas traducciones de Sentiñón, una sobre la obra de fisiología popular Conócete a ti mis$m o$, a la que incorpora varios apéndices y numerosas anotaciones, elevando su interés científico; la segunda, la conocida novela del egiptólogo Ebers, La hija del rey de Egipto, relato de gran éxito por el número de reediciones ${ }^{73}$. En 1882 se editó otra traducción suya del alemán, el libro Germania, de J. Scherr.

Su participación social se amplió con el Ateneo Barcelonés, uno de los principales focos culturales de la Barcelona burguesa de esta época que proviene de la fusión entre el Centro Mercantil Barcelonés y el Ateneo Catalán. Letamendi había formado parte de la Junta Directiva del Ateneo Catalán y lo fue también en la primera de la sociedad fusionada. Sentiñón fue socio del Ateneo Barcelonés, al menos desde los inicios de la década de los ochenta ${ }^{74}$, y participó en él de forma reseñable hasta su fallecimiento ${ }^{75}$. Sus actuaciones en este centro fueron de tres tipos: como profesor de

73 LITVAK (1990), p. 204 y 244, n. 14. Esta autora atribuye erróneamente la traducción a J. Ramón Mélida.

74 Ateneo Barcelonés, Barcelona. Libro de Registro de Socios del Ateneo Barcelonés (1882-1889), p. 254. No consta el año de inscripción.

75 Ateneo Barcelonés, Barcelona. Libro de Residentes, p. 332. Sentiñón figura con el nº 73; la baja es de diciembre de 1902, el mes y año de su defunción. 
idiomas, como conferenciante y como miembro muy activo de la Sección de Ciencias Exactas.

Su trabajo como profesor de idiomas comenzó en $1883^{76}$ y consta que sus clases de ruso eran especialmente apreciadas ${ }^{77}$. En el curso 1885-1886 el Ateneo dotó una cátedra de alemán y puso al frente a Sentiñón ${ }^{78}$.

Como miembro de la Sección de Ciencias Exactas se presentó a vocal directivo en mayo de $1884^{79}$, aunque el candidato elegido resultó ser el también médico Emerenciano Roig Bofill. En el año 1886 la Sección, motivada por la epidemia de cólera, organizó varias sesiones de debate sobre la higiene pública en Barcelona, en las que participó activamente ${ }^{80}$. Fruto de estos debates varios miembros, entre ellos los médicos Rodríguez Méndez, Roig Bofill y Sentiñón, propusieron crear un colectivo que bajo la denominación de Liga Sanitaria de Barcelona pusiese su influencia y capacidad al servicio de los intereses sanitarios de la ciudad. La propuesta fue bien acogida, adhiriéndose más de un centenar de socios, con lo que el 21 de mayo de ese año se constituyó la Junta Directiva, en la que figura como tesorero ${ }^{81}$. La Liga Sanitaria de Barcelona, que no era una sociedad médica puesto que aceptaba a todos los interesados por la defensa de la salud que supieran leer y escribir, recogía entre sus objetivos la promoción de las instituciones que protegiesen la salud del proletariado.

En el mismo sentido, otra actuación de la Sección que contó con su participación fue el debate organizado en el curso 1889-90 sobre la necesidad de modificar la legislación limitadora del trabajo infanti1 ${ }^{82}$.

En cuanto a las conferencias impartidas, cabe destacar la relativa a las acciones de la población para preservarse de la viruela ${ }^{83}$, celebrada en el año 1884 y que resultó tan polémica como su libro sobre esta enfermedad. También la dedicada a «Sofía de Kovalevsky, su vida y sus recuerdos de George Eliot», que tuvo lugar el 10 de febrero de 1893, en la que abogó por la formación de la mujer como elemento necesario para elevar su condición social en España ${ }^{84}$.

Pero su presencia en los actividades del Barcelonés no supone su única actividad social de carácter público. En la década de los ochenta el grupo más radical de librepensadores se había organizado en torno al filántropo y masón Rosendo Arús y Arderiu y su Círculo La Luz, que desde 1885 editaba el periódico La Luz. El Círculo se

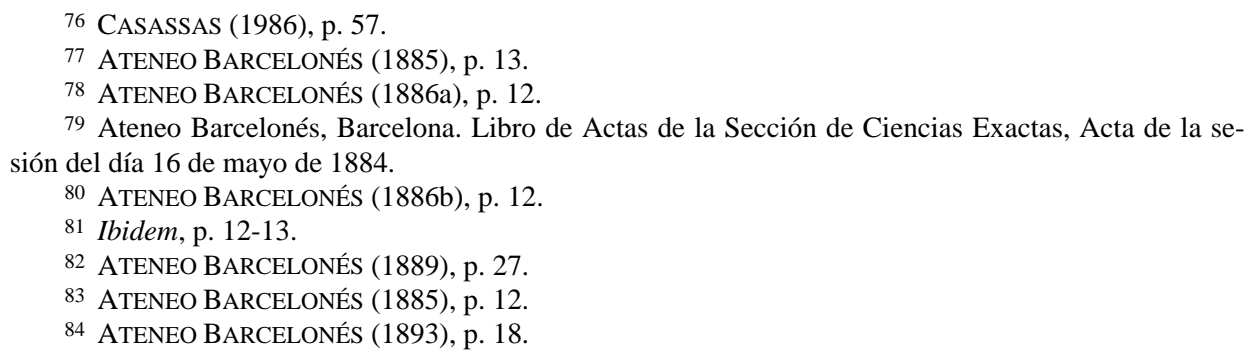


convirtió en un centro de convivencia de librepensadores, republicanos, masones y anarquistas, al que acudieron a colaborar destacados libertarios como Tarrida del Mármol, López Montenegro, Sentiñón y Anselmo Lorenzo. El periódico La Luz publicó habitualmente noticias anarquistas, así como textos breves de Bakunin y Guillaume, pero en sus 25 números no hemos encontrado la firma de Sentiñón, por lo que para conocer sus actuaciones recurrimos a los escritos del anarquista Adrián del Valle ${ }^{85}$, participante del Círculo en su juventud. Por él sabemos que frecuentaba el local, donde impartía gratuitamente clases de inglés y disertaba como conferenciante sobre higiene; también colaboraba en el periódico, aunque de forma anónima.

Paralelamente al desarrollo del Círculo La Luz, en enero de 1886 salió una nueva publicación libertaria, Acracia, caso singular por su calidad y contenidos $^{86}$, que finalizó en junio de 1888. Esta revista tuvo el impulso de Farga Pellicer y Anselmo Lorenzo ${ }^{87}$; Sentiñón también colaboró, aunque según la pauta casi general no firmaba sus artículos o lo hacía con alguna de sus iniciales. Palmiro de Lidia le ha atribuido numerosas traducciones ${ }^{88}$, aunque no es difícil adjudicarle varios artículos de contenido médico que responden a los temas de su interés y que firmaba con alguna de sus iniciales; también son suyas las noticias médicas que tienen casi todos los números de esta revista.

Del mayor interés es un artículo de contenido ideológico que firmó con las iniciales de su primer nombre y de los dos apellidos ${ }^{89}$, se trata de la respuesta a un texto del anarquista Teobaldo Nieva, en la que Sentiñón expone la diferencia entre trabajador y obrero, desecha los conceptos confusos de Nieva sobre colectivismo, comunismo y producto íntegro, pero, sobre todo, explica su idea de la revolución, basada en el trabajo orgánico y las propuestas ideológicas y opuesta al uso innecesario de la fuerza.

Desde las páginas de Acracia se anunció un nuevo diario anarquista, El Productor, considerado como la publicación obrera más importante de su tiempo ${ }^{90}$, pese a que pronto pasó a semanario por problemas económicos. Sentiñón participó ampliamente en este periódico, encargándose de las noticias extranjeras y científicas, así como de escribir artículos políticos ${ }^{91}$, muy difíciles de identificar por ser anónimos.

La aparente contradicción entre el intelectual anarquista y librepensador y el médico que participaba activamente en la Barcelona burguesa del Ateneo es la imagen real de la dualidad de ambientes en los que actuaba Sentiñón.

\footnotetext{
85 Se trata de la serie de cuatro artículos que con la denominación de «Evocando al pasado» publicó, bajo su pseudónimo habitual de Palmiro de Lidia, en La Revista Blanca del 15 julio al 15 de septiembre de 1927. Con respecto a Sentiñón hay datos interesantes en los dos primeros.

86 ABELló (1987), p. 37.

87 BUENACASA (1977), p. 157.

88 LIDIA (1927), p. 140.

89 L.S.C. (1886).

90 ArbeloA (1970), p. 165.

91 LIDIA (1927), p. 117.
} 
También la década de los ochenta resultó la más productiva como escritor. El profesor de la Facultad de Medicina de Barcelona, de la que fue decano posteriormente, Juan Giné y Partagás, convocó el Primer Certamen Frenopático durante los días 25 al 28 de septiembre de 1883. En su cuarta sesión se presentó una extensa «Memoria acerca de los manicomios en España», elaborada por el médico neoyorquino G. Seguin y traducida por Gaspar Sentiñón para su lectura en este congreso. Ese mismo año salió su segundo libro, El cólera y su tratamiento, con un doble destino: profesionales y población general; en el año siguiente, se presentaba el tercero y último de sus libros, La viruela y su tratamiento curativo, preservativo y exterminativo, con una línea higienista similar al anterior, pero más radical en la responsabilidad que atribuye a los médicos en el estado de esta enfermedad en España.

También en 1884 nació en Barcelona su única hija María Sentiñón Gatell. Sabemos que estaba casado con María Gatell Sentís, nacida en 1853 en Cornudella ${ }^{92}$, provincia de Tarragona. En 1885 se editó una obra de especial importancia, la Morfología general de los organismos, de Haeckel; Sentiñón había colaborado en la traducción con Salvador Sanpere y Miquel y también se encargó de la revisión general del libro y de completar las abundantes ilustraciones para la editorial Barris de Barcelona.

En el periodo 1887-88 se publicó su traducción de un nuevo volumen de medicina, el Manual práctico de oftalmología, de Michel, pero, sobre todo, hay que destacar un acontecimiento fundamental para la ciudad de Barcelona: la Exposición Universal de 1888. En este marco se organizaron los Congresos de Ciencias Médicas, celebrados la semana del 9 al 15 de septiembre. Sentiñón participó con el tema titulado «Estado actual de la lepra en España y medios para evitar su difusión». En este trabajo de orientación histórica basa sus objetivos preventivos en la necesidad de informar adecuadamente al proletariado rural. En 1891 se publicó su traducción en dos volúmenes de un importante texto de medicina legal, el de Hofmann.

En 1895, Rodríguez Méndez se hizo eco en su revista de una nueva actividad de Sentiñón, en la que combina su vocación de higienista con la utilidad comercial: había abierto un local de venta y exposición de aguas mineromedicinales. La exposición, de la que se anunciaba un catálogo, presentaba una rica variedad de aguas minerales de España y otros países, cuya garantía de autenticidad era, a juicio de Rodríguez Méndez, el nombre de Sentiñón, del que, entre calificativos de admiración, recordaba sus relaciones en toda la Europa médica ${ }^{93}$.

En el mes de agosto de 1897 se celebró el XII Congreso Internacional de Medicina en Moscú, por ello se había constituido un Comité Nacional presidido por Bartolomé Robert, con Salvador Cardenal de vicepresidente y Gaspar Sentiñón y Joaquín Durán

\footnotetext{
92 Archivo Parroquial, Cornudella. Libro de bautismos, 7, f. 409r.

93 RODRÍGUEZ MÉNDEZ (1895), p. 589-590.
} 
de secretarios. No consta en las Actas del Congreso que Sentiñón presentase ningún trabajo, ni que acudiese a Moscú ${ }^{94}$. Su elección debió estar influenciada tanto por su conocimiento de la medicina internacional como por su dominio del idioma ruso.

En 1900 se publicó su traducción del español al inglés del libro dirigido por Sierra sobre Méjico y en 1902 se editó el último libro relacionado con Sentiñón, la Enciclopedia de ginecología, de Veit, de la que fue uno de los traductores.

El 13 de febrero de ese mismo año Sentiñón participó como miembro del jurado calificador en la elección de textos para niños sobre educación integral, lectura enciclopédica e higiene pública y privada, respectivamente, en el marco del Certamen de Pedagogía Popular que organizó la Cooperativa Intelectual de Barcelona-Gracia ${ }^{95}$. Este tipo de certámenes se instituían para dotar de material pedagógico a la naciente Escuela Moderna de Ferrer Guardia ${ }^{96}$. Con él formaron parte del jurado Rodríguez Méndez, Giné y Partagás, Salas Antón, Odón de Buen, Clemencia Jacquinet y Anselmo Lorenzo, los tres últimos miembros de la Escuela Moderna.

En el entorno familiar, el 28 de noviembre de 1902 falleció su hija a causa de una meningitis tuberculosa, a los 18 años de edad ${ }^{97}$. El periódico El Diluvio y la Revista de ciencias médicas de Barcelona atribuyeron a esta dolorosa pérdida el agravamiento del estado de salud de Sentiñón, que murió pocos días más tarde, el 12 de diciembre de 1902, a causa de una uremia por nefritis crónica.

Su muerte no pasó desapercibida y recibió las correspondientes necrologías de Gaceta médica catalana ${ }^{98}$, de la Revista de ciencias médicas de Barcelona ${ }^{99}$ y de la Revista balear de ciencias médicas ${ }^{100}$, en la que se reproducían sus noticias médicas.

Desde la prensa no profesional, el republicano El Diluvio le dedicó una amplia nota ya comentada, en la que repasaba las principales actuaciones de su vida. También los periódicos libertarios recogían el fallecimiento, como la publicación madrileña Tierra y Libertad ${ }^{101}$ y la barcelonesa La Huelga General. Esta última nos aporta alguna de las claves de su actividad profesional: «Dedicado incesantemente al trabajo y al estudio, explotado sin piedad por editores y doctores, unos enriqueciéndose a su costa y otros pavoneándose con las galas de la sabiduría de aquel maestro de maestros,...» ${ }^{102}$, lo que coincide con parte de las ideas expresadas por la nota de la tan distinta Gaceta médica catalana: «Con sus vastos conocimientos ha sido el Mentor de algunos maestros y de otros tenidos como eminencias, el refugio de las dudas, de

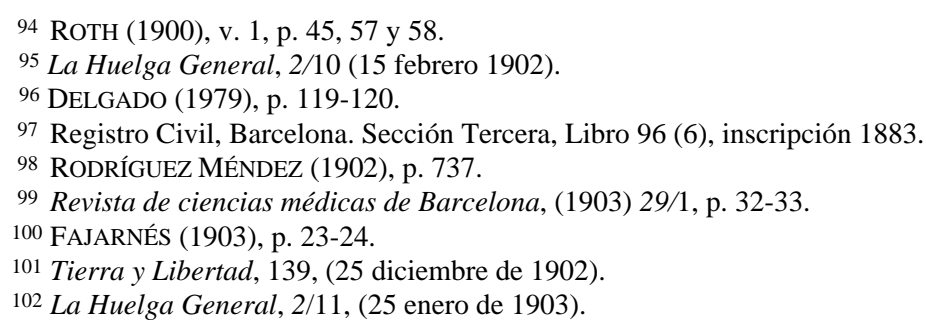




\section{JOSÉ VICENTE MARTÍ BOSCÀ}

las flaquezas y deficiencias de científicos de toda suerte y de casi todas las nacionalidades» ${ }^{103}$. Un detallado estudio de su obra médica nos permitirá ubicarlo correctamente en el desarrollo de la medicina en la España de la Restauración.

\section{REFERENCIAS BIBLIOGRÁFICAS}

ABELló, T. (1987), Les associacions obreres i la cultura, L'Avenç, 104, 36-39.

Álvarez JunCO, J. (1971), La Comuna en España, Madrid, Siglo XXI.

ARbElOA, V.M. (1970), La prensa obrera en España, I (1869-1899), Revista del trabajo, 30, 117-195.

ArbeloA, V.M. (1972), I Congreso Obrero Español, Ed. V. M. Arbeloa, Madrid.

AtENEO BARCELONÉS (1885), Acta de la sesión pública del Ateneo Barcelonés celebrada el día 29 de Diciembre de 1884, Barcelona, Establecimiento Tipográfico Sucesores de N. Ramírez.

Ateneo BARCElonés (1886a), Acta de la sesión pública del Ateneo Barcelonés celebrada el día 18 de Diciembre de 1885, Barcelona, Establ. Tip. Suc. de N. Ramírez.

Ateneo BARCElonés (1886b), Acta de la sesión pública del Ateneo Barcelonés celebrada el día 22 de Noviembre de 1886, Barcelona, Establ. Tip. La Academia.

AtENeo BARCElonés (1889), Acta de la sesión pública del Ateneo Barcelonés celebrada el día 5 de Noviembre de 1889, Barcelona, Establ. Tip. Casa de la Caridad.

Ateneo BARCElonés (1893), Acta de la sesión pública del Ateneo Barcelonés celebrada el día 14 de Diciembre de 1893, Barcelona, Establ. Tip. Casa de la Caridad.

BAKunin, M. (1973-82), Oeuvres complètes de Bakounine, 7 vols., Paris, Ed. Champ Libre.

BAKUnin, M. (1977-86), Obras completas, 5 vols., Madrid, Ed. La Piqueta,.

Bretau, F. (1987), Aportaciones a la toxicología del Dr. Gaspar Sentiñón y Cerdaña, Gimbernat, 8, 227-236.

BÜCHNER, L. (1873), Ciencia y naturaleza. Ensayos de filosofía y de ciencia natural, Málaga, 2v, Hijos de J. G. Taboadela.

BUENACASA, M. (1977), El movimiento obrero español 1886-1926, Madrid, Ed. Júcar.

CAlbet, J.M.; CoRbella, J. (1981-83), Diccionari biogràfic de metges catalans, 3 vols., Barcelona, Ed. Universitat de Barcelona.

CARreras, A. (1979), La dermatologia en la medicina catalana. Contribució a la medicina mundial. Barcelona, Ed. Real Academia de Medicina de Barcelona.

Casassas, J. (1986), L'Ateneu Barcelonès. Dels seus orígens als nostres dies, Barcelona, Ed. de la Magrana-Institut Municipal d'Història.

CONSEIL GÉNÉRAL (1974), Le Conseil Général de la Première Internationale 1868-1870. Procès-verbaux, Moscou, Éditions du Progrès.

103 RodRíGUEZ MÉNDEZ (1902), p. 737. Subrayado en el original. 
Corbella, J; Calbet, J.M. (1969), Introducción al estudio de la obra médica y política de Gaspar Sentiñón, Asclepio, 21, 135-155.

DAY, GE (1880), El dolor de cabeza. Su naturaleza, sus causas y tratamiento, Barcelona, Imprenta barcelonesa.

Delgado, B. (1979), La Escuela Moderna de Ferrer i Guardia, Barcelona, Ed. CEAC.

EBERS, J. (1881), La hija del rey de Egipto, 2 vols., Barcelona, Tipo-lit. de C. Verdaguer.

FAJARNÉS, E. (1903), «Necrología. El Dr. Sentiñón», Revista balear de ciencias médicas, 24/1, 23-24.

FIGUIER, L. (1881), Conócete a ti mismo, tratado popular de fisiología humana, 2v, Barcelona, Imprenta y librería religiosa y científica del heredero de Pablo Riera.

Freymond, J. (1973), La Primera Internacional, 2 vols., Madrid, Ed. Zero.

GINÉ y PARTAGÁs, J. (1898), Discurso inaugural leído en la solemne apertura del curso académico de 1898-99 ante el claustro de la Universidad de Barcelona. Barcelona, Imprenta de Henrich y $\mathrm{C}^{\mathrm{a}}$.

GONZÁLEZ Sugrañes, M. (1896), La República en Barcelona, Barcelona, Imprenta de Henrich y $\mathrm{C}^{\mathrm{a}}$.

GUERARD, Dr. de (1879), Los dientes. Su estructura y desarrollo, su higiene y sus enfermedades, Barcelona, Imprenta y librería religiosa y científica del heredero de D. Pablo Riera.

Guillaume, J. (1985), (facsímil de 1905-1910) L'Internationale. Documents et souvenirs, 2 vols., Paris, Ed. Gérard Lebocini.

HAECKEL, E. (1885), Morfología general de los organismos, Barcelona, Barris y Compañía, Editores.

HERNÁNDEZ DíAZ, J.M. (1992), «Influencias europeas en las propuestas educativas de la Primera Internacional en España», Historia de la Educación, 11, 205-230.

Hofmann, E.R. von (1891), Tratado de medicina legal, $2^{\text {a }}$ ed., 2 vols., Madrid, Administración de la Revista de medicina y cirugía prácticas.

Kraus, L.A. (1872), Manual completo de terapéutica para médicos prácticos y cirujanos, Barcelona, Establ. Tip. de Narciso Ramírez y Compañía.

LETAMENDI, J. (1894), Curso de clínica general, o canon perpetuo de la práctica médica para el uso de estudiantes y aun de médicos jóvenes, 2 vols., Madrid, Imprenta de los sucesores de Cuesta.

LIDA, C.E. (1972), Anarquismo y Revolución en la España del XIX, Madrid, Ed. Siglo XXI.

LIDA, C.E. (1973), Antecedentes y desarrollo del movimiento obrero español (1835-1888), Madrid, Ed. Siglo XXI.

LiDIA, P. de (1927), «Evocando al pasado», La Revista Blanca, 6/100, 115-118; 6/101, 138$142 ; 6 / 103,210-214$ у $6 / 104,245-249$.

LIMA, S. de M. (1892), O socialismo na Europa, Lisboa, Typ. da Companhia Nacional Editora.

LiTVAK, L. (1990), España 1900. Modernismo, anarquismo y fin de siglo. Barcelona, Anthropos.

LóPEZ PiÑERO, J.M. (1992), Las ciencias médicas en la España del siglo XIX, Ayer, 7, 193-240. 


\section{JOSÉ VICENTE MARTÍ BOSCÀ}

LÓPEZ PiÑERO, J.M. et al. (1983), Diccionario histórico de la ciencia moderna en España, 2 vols., Barcelona, Ed. Península.

LORENZO, A. (1974), El proletariado militante, Madrid, Ed. Zero.

MAdRID SANTOS, F. (1995), «Gaspar Sentiñon e la Prima Internazionale spagnola (18681871)», Rivista storica dell'anarchismo, 2/2, 37-52.

Martí Boscà, J.V. (1997), Medicina y sociedad en la vida y la obra de Gaspar Sentiñón Cerdaña (1835-1902), microfichas, València, Ed. Universitat de València.

Michel, J. (1887-1888), Manual práctico de oftalmología, Madrid. Administración de la Revista de medicina y cirugía prácticas.

NetTlau, M. (1925), Miguel Bakunin, la Internacional y la Alianza en España (1868-1873), Buenos Aires, Ed. La Protesta.

Nettlau, M. (1926), «Un poco de historia. Alrededor de Miguel Bakounine y Gaspar Sentiñón», La Revista Blanca, 4/83, 327-328.

Nettlau, M. (1930), Documentos inéditos sobre la Internacional y la Alianza en España, Buenos Aires, Ed. La Protesta.

NetTlau, M. (1969), La Première Internationale en Espagne (1868-1888), Dordrecht, D. Reidel Publishing Co.

NiEMEYER, P. (1879), La tos y los resfriados. Su origen, tratamiento y prevención, Barcelona, Imprenta y librería religiosa y científica del heredero de D. Pablo Riera.

NúÑEZ RUIZ, D. (1975), La mentalidad positiva en España: desarrollo y crisis, Madrid, Ed. Tucar.

REVEnTós, M. (1987), Els moviments socials a Barcelona en el segle XIX, Barcelona, Ed. Crítica.

RiBAS, P. (1979), «Las relaciones entre el socialismo alemán y el español», Estudios de historia social, 8-9, 227-286.

RichARD, A. (1896), «Bakounine et l'Internationale a Lyon, 1868-1870», Revue de Paris, 119-160.

RoBin, P. (1981), Manifiesto a los partidarios de la educación integral, Barcelona, Ed. José J. de Olañeta.

RODRÍGUEZ MÉNDEZ, R. (1881), «Bibliografía», Revista de medicina y cirugía prácticas, 9/25, 211.

RodRíGUEZ MÉNDEZ, R. (1895), Depósito general de aguas minero-medicinales, Gaceta médica catalana, 18/438, 589-590.

RODRÍGUEZ MÉNDEZ, R. (1902), «iSentiñón!», Gaceta médica catalana, 25/611, 737.

RODRÍGUEZ MÉNDEZ, R. (1919), «Historia de la Gaceta médica catalana», Gaceta médica catalana, 40/1000, 170.

Roth, W. (comp.) (1900), Comptes-rendus du XII Congrès International de Médicine, Moscou, Typ.-lit. Societé I. N. Kouchnérev \&C-ie,

ROVIRA, R. (1884), «Sección bibliográfica», La Independencia médica, 15, 365. 


\section{BIOGRAFÍA DE GASPAR SENTIÑÓN CERDAÑA: DATOS Y ENIGMAS DE UN INTRODUCTOR}

SÀnCHEZ i Ferré, P. (1990), La maçoneria a Catalunya (1868-1936), Barcelona, Ed. Ajuntament de Barcelona-Edicions 62.

SCHERR, J. (1882), Germania. Veinte siglos de historia bajo el punto de vista de la civilización. 2 t. en 1 vol, Barcelona, L. Tasso Serra.

Seco Serrano, C. (ed.) (1969), Actas de los Consejos y Comisión Federal de la Región Española (1870-1874), v 1, Barcelona, Ed. Universidad de Barcelona.

SENTiÑón, G. (1880), La digestión y sus tropiezos según las investigaciones químicas y fisiológicas más recientes, Barcelona, Imprenta y librería religiosa y científica del heredero de D. Pablo Riera.

SENTIÑóN, G. (1883), El cólera y su tratamiento, Barcelona, Imprenta barcelonesa.

SENTIÑ́́N G. (1884), La viruela y su tratamiento curativo, preservativo y exterminativo, Barcelona, Imprenta barcelonesa.

L.S.C. (SEntiñón, G.) (1886), Comunicación importante, Acracia, 1/9, 96-99.

SEnTiÑón, G. (1897), «¡José de Letamendi!», Gaceta médica catalana, 20/481, 421.

SIERRA, J. (dir.) (1900-1904), Mexico: its social evolution;..., 3 vols., México, J. Ballesca \& co.

TERmes, J. (1977), Anarquismo y sindicalismo en España, Barcelona, Ed. Crítica.

VeIT, J. (dir.) (1902), Enciclopedia de ginecología, 4 vols., Madrid, Administración de la Revista de medicina y cirugía prácticas.

Vilanou, C. (1978), Anarquismo, educación y librepensamiento en Cataluña, 1868-1901, tesis de licenciatura inédita, Universidad de Barcelona. 
